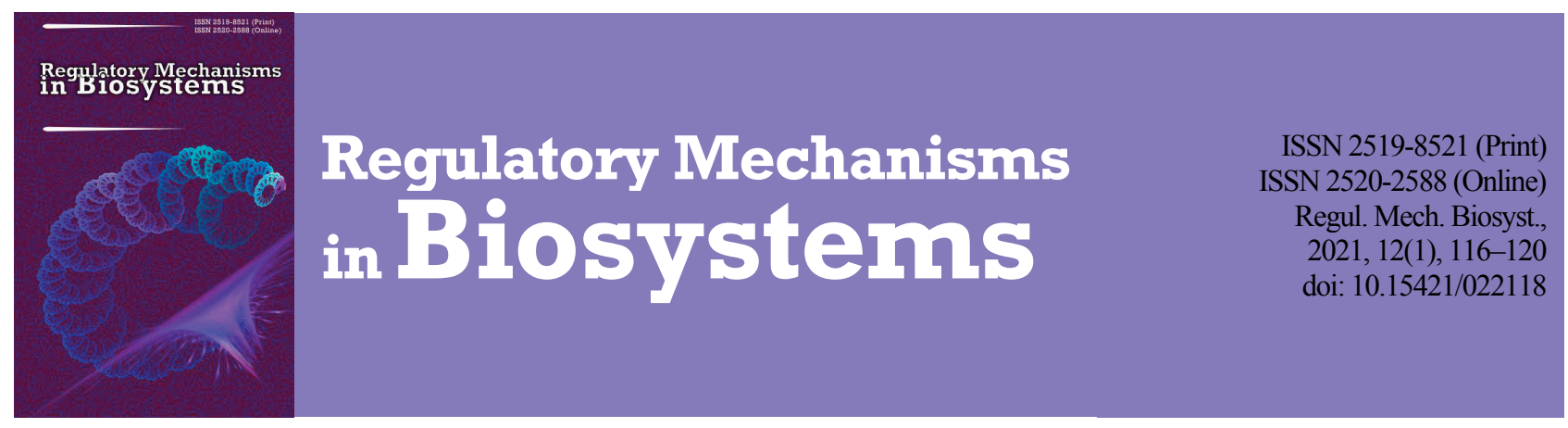

\title{
Effect of ozonation on resistance of ovine and human erythrocytes to hypothermic storage
}

\author{
K. M. Holovina, O. M. Bobrova, S. Y. Kovalenko, Y. S. Hovorova, O. A. Nardid \\ Institute for Problems of Cryobiology and Cryomedicine of the National Academy of Sciences of Ukraine, Kharkiv, Ukraine
}

Article info

Received 20.01.2021

Received in revised form 25.02.2021

Accepted 27.02.2021

Institute for Problems of Cryobiology and Cryomedicine of the National Academy of Sciences of Ukraine, Pereyaslavska st., 23 Kharkiv, 61016, Ukraine. Tel.: +38-057-373-41-43. E-mail: helen.bobrova.77 @gmail.com

\begin{abstract}
Holovina, K. M., Bobrova, O. M., Kovalenko, S. Y., Hovorova, Y. S., \& Nardid, O. A. (2021). Effect of ozonation on resistance of ovine and human erythrocytes to hypothermic storage. Regulatory Mechanisms in Biosystems, 12(1), 116-120. doi:10.15421/022118
\end{abstract}

\begin{abstract}
Long-term hypothermic storage of animal blood can lead to the loss of its quality and can cause complications in recipient animals after transfusion, so the search for new methods of increasing the preservation of erythrocytes after hypothermic storage continues. The article presents the data of the ozonation effect on the preservation rate of ovine and human erythrocytes during hypothermic storage with Alsever's solution and mannitol medium. Hemolysis, osmotic fragility and distribution density of ovine and human erythrocytes by the sphericity index were determined at different stages of hypothermic storage. The ovine erythrocytes in the control had a lower osmotic resistance compared to human erythrocytes; however, their osmotic fragility did not change significantly after hypothermic storage for 8 weeks, and for human erythrocytes, it significantly increased. Storage of ovine and human erythrocytes longer than 8 weeks led to a sharp hemolysis, while the level of hemolysis of ovine erythrocytes was lower than that of human erythrocytes. Preservation of ozonated erythrocytes is higher than non-ozonated ones during prolonged hypothermic storage. The effect of ozonation on the preservation rate of red blood cells depended on the composition of the preservation media. Hypothermal storage of human erythrocytes in Alsever's solution for up to 8 weeks led to a shift in the density of distribution according to the sphericity index towards spheroidization of cells; in a medium with mannitol, the number of flattened cell forms increased. After 8 weeks of hypothermic storage of ovine erythrocytes, most of the cells had high sphericity indices. Pretreatment of human and sheep erythrocytes with ozone allows the distribution of cells to be kept closer to the control during long-term storage, which is especially pronounced in mannitol medium. Ovine erythrocytes retained the ability to form rosettes with human T-lymphocytes after hypothermic storage for up to 12 weeks. Thus, ozone pretreatment and the use of mannitol as part of hypothermic storage medium could be an approach to improve the quality of preserved ovine erythrocytes.
\end{abstract}

Keywords: red blood cells; Alsever's solution; mannitol; preservation medium; osmotic fragility; sphericity index; rosette formation.

\section{Introduction}

The first case of a blood transfusion in an animal was recorded in 1665, when Richard Lower transfused canine blood (Kumar, 2017). Over the past century, blood transfusions in veterinary medicine have achieved a considerable success and now transfusion therapy has become an integral part of emergency veterinary medicine (Yagi, 2016; Le Gal et al., 2020; Martinez-Sogues et al., 2020). The most common blood banks are established for dogs and much less for cats. There are no blood banks for other species of animals, and when it is urgently needed for an animal, rapid selection and screening of a donor becomes a serious problem. Transfusion therapy is widely used in treatment of farm animals (Durham, 1996; Bell, 2006; Saritha et al., 2016). However, animals have numerous blood groups (Ryskina \& Gilmiyarova, 2015; Webb, 2019) and the necessity to check animals for the presence of blood-parasitic and infectious diseases (Wardrop et al., 2005; Davidow, 2013) makes donor selection a difficult process. This necessitates the establishment of blood banks not only for dogs and cats, but also for farm animals.

Ovine blood is used by veterinarians not only for transfusion therapy (Wain et al., 1985; Chandler et al., 1998; Sousa et al., 2014), but also by scientists for modeling transfusion conditions (Simonova et al., 2014; Fung et al., 2016) because in the animals the anatomy, hemodynamic and microcirculatory characteristics and red blood cells' lifespan are very similar to those of humans. Ovine red blood cells are also used in immunological studies to test the rosette reactions with T lymphocytes (Wybran et al., 1972; Ladics, 2007; McAllister et al., 2017).
Long-term hypothermic storage of animal blood can lead to loss of quality and can cause complications in recipient animals after transfusion. Sousa et al. (2014) showed that in whole blood of sheep after storage for 35 days there was a progressive increase of hemoglobin, potassium and lactate concentration in blood plasma and decrease of sodium, bicarbonate and glucose concentration. The morphological changes, metabolic disorders, and oxidative damage that occur during storage have a negative effect on viability and function of erythrocytes, reduce their survival after transfusion (Kisielewicz \& Self, 2014). Transfusion of such blood can lead to the most common responses in ruminant mammals such as tachycardia, tremor, fever, itching, dyspnea, hematuria and hemoglobinuria (Sousa et al., 2014). It was shown that the rate of spontaneous hemolysis of human red blood cells during hypothermic storage decreases after ozone treatment (Zinchenko et al., 2012).

Ovine erythrocytes have a number of features in comparison with those of humans and other mammals. It is known that deformability of erythrocytes is size and shape-dependent. Erythrocytes of pig, hamster, rat, mouse, and rabbit are more deformable than those of sheep, horse, elephant, and dog. The deformity is characterized by erythrocytes' elongation and aggregation (Saganuwan, 2019). Erythrocytes group together to form the chains named rouleaux (French for rolls) (Shinbrot, 2019). There is a diversity in erythrocyte aggregability among the mammalian species (Windberger et al., 2017). For example, erythrocyte aggregation is high in humans, and it is very low in cows, sheep and others (Windberger et al., 2017). Erythrocytes of sheep have lower values of elongation index within the higher shear stress range. Such a behaviour may reflect the exception- 
ally small size of ovine erythrocytes compared to other species (elephant, horse, dog and others) (Baskurt et al., 2007).

The aim of this work was to comparatively analyze the ozonation effect on sensitivity of ovine and human erythrocytes to long-term hypothermic storage in different media.

\section{Materials and methods}

Blood of sexually mature sheep males (Ovis aries Linnaeus, 1758) was collected from the external jugular vein and prepared with $3.8 \%$ sodium citrate. The experiments were carried out in accordance with the Law of Ukraine "On the Protection of Animals Against Cruelty" (No. 3447-IV of 02.02.2006) in compliance with the requirements of the Committee in Bioethics of the Institute for Problems of Cryobiology and Cryomedicine of the National Academy of Sciences of Ukraine, in accordance with the provisions of the European Convention on the Protection of Vertebrates, Used for Experimental and Other Scientific Purposes" (Strasbourg, 1986).

Donor blood (male, A (II) +) was received at the Kharkiv Regional Center for Blood Service. The blood was centrifuged at $800 \mathrm{~g}$ for $10 \mathrm{~min}$, the plasma was removed and then erythrocytes were three times washed by centrifugation under the same conditions in a 10-time volume of isotonic $\mathrm{NaCl}$ solution containing $0.01 \mathrm{M}$ Tris buffer (pH 7.2). The erythrocyte precipitate was divided into two parts. One part was the control group (non-ozonated), the other part was ozonated (ozone concentration was $120 \mu \mathrm{g} / \mathrm{mL}$ ). Ozone was obtained by means of electrosynthesis, when oxygen gas passes through a barrier-type ozonator. Then the ozonated and non-ozonated erythrocytes were re-suspended with an equal volume of preservation medium: Alsever's solution ( $20.5 \mathrm{~g} / \mathrm{L}$ glucose, $8 \mathrm{~g} / \mathrm{L}$ sodium citrate, $0.552 \mathrm{~g} / \mathrm{L}$ citric acid, $4.2 \mathrm{~g} / \mathrm{L}$ sodium chloride in distilled water) or mannitol medium (50 g/L mannitol, $0.9 \mathrm{~g} / \mathrm{L}$ sodium chloride in distilled water). Antibiotic Cifran (Yuriya-farm, Ukraine) was added to all suspensions at a final concentration $0.01 \mathrm{mg} / \mathrm{mL}$. The erythrocytes were stored in the refrigerator at temperatures of $2 \ldots 4^{\circ} \mathrm{C}$.

The hemolysis level of erythrocytes was spectrophotometrically determined at $543 \mathrm{~nm}$ wavelength, which was expressed as a percentage in respect of $100 \%$ hemolysis (added Triton X-100 at a concentration of $0.1 \%$ ). The osmotic fragility of red blood cells was assessed by the release of hemoglobin from the cells in sodium chloride solutions with different tonicity $(0-150 \mathrm{mM}), \mathrm{pH}=7.4$. The distribution density of erythrocytes by the sphericity index was determined by the method of low-angle light scattering (Gordiyenko et al., 2004). The change in the distribution of erythrocytes by the sphericity index indicates a change in the quantitative ratio of various cell forms in the population of erythrocytes (Lomako et al., 2012; Ostras et al, 2019).

To assess the ability of ovine erythrocytes to participate in the rosette formation after hypothermic storage we used human lymphocytes (Wybran et al., 1972; Klaus, 1990). Lymphocytes were isolated from donor blood with a shelf life up to 4 days in a density gradient (ficoll-triombrast) and their viability was evaluated (trypan blue eosin staining), then a concentration of 2 million cells in $\mathrm{ml}$ was prepared. Ovine erythrocytes were added to lymphocyte suspension (erythrocyte:lymphocyte ratio should not exceed 50:1). The mixture was incubated in thermostat $\left(37^{\circ} \mathrm{C}\right)$ for 10 minutes. Cells were stained according to Romanowsky-Giemsa and then the rosette formation was observed using microscope "Olympus IX 70" (USA). The data in figures and tables are given as mean \pm standard deviation. Statistical processing was performed using ANOVA with Bonferroni correction. Data were averaged over at least 5 experiments. The level of statistical significance was assumed as $\mathrm{P}<0.05$.

\section{Results}

The level of hemolysis of ovine erythrocytes in control and after oneweek storage did not significantly differ for all studied preservation media (Table 1). The ozonation treatment also did not change this index. During hypothermic storage we observed an increase of ovine erythrocyte hemolysis. After 6 weeks of storage, there was a strong difference in hemolysis between ozonated and non-ozonated erythrocytes. Our results indicate that starting from the 6th week of storage the erythrocyte hemolysis in mannitol solution was significantly lower than in the Alsever's solution. From the week 8 to 12 of storage, the level of erythrocyte hemolysis sharply increased in both preservation media; however, in mannitol solution this index is still significantly lower compared to the Alsever's solution. In addition, it can be noted that starting from week 6 of storage, the level of hemolysis of ozonated erythrocytes was smaller than for non-ozonated, the lowest values of hemolysis were observed in the medium with mannitol for ozonated erythrocytes for all the terms of observation.

\section{Table 1}

Hemolysis (\%) of ovine red blood cells (RBC) during hypothermic storage depending on the preservation medium and ozone pretreatment

\begin{tabular}{ccccc}
\hline \multirow{2}{*}{$\begin{array}{c}\text { Storage } \\
\text { time, weeks }\end{array}$} & \multicolumn{2}{c}{ RBC in the Alsever's solution } & \multicolumn{2}{c}{ RBC in mannitol medium } \\
\cline { 2 - 5 } & non-ozonated & ozonated & non-ozonated & ozonated \\
\hline 0 & $0.28 \pm 0.09$ & $0.39 \pm 0.12$ & $0.26 \pm 0.08$ & $0.30 \pm 0.10$ \\
1 & $2.32 \pm 0.18$ & $2.01 \pm 0.22$ & $1.80 \pm 0.20$ & $1.72 \pm 0.21$ \\
6 & $8.41 \pm 0.49$ & $6.45 \pm 0.41^{\#}$ & $8.12 \pm 0.45$ & $8.01 \pm 0.49^{*}$ \\
8 & $16.22 \pm 1.06$ & $12.19 \pm 0.68^{\#}$ & $12.83 \pm 0.99^{*}$ & $10.09 \pm 0.87^{* \#}$ \\
12 & $28.63 \pm 1.50$ & $27.01 \pm 1.05$ & $21.14 \pm 1.42^{*}$ & $17.56 \pm 1.24^{* \#}$ \\
\hline
\end{tabular}

Note: * - significant differences compared to the Alsever's solution $(\mathrm{P}<0.05),{ }^{\#}$ significant differences compared to non-ozonated cells $(\mathrm{P}<0.05) ; \mathrm{n}=5$.

The hemolysis level of human erythrocytes increases faster with duration of hypothermic storage, compared with ovine erythrocytes (Table 2). As for ovine erythrocytes, hemolysis of human erythrocytes is lower in a mannitol medium. From week 6 of storage a higher preservation of ozonated red blood cells compared with non-ozonated ones can be noted.

\section{Table 2}

Hemolysis (\%) of human red blood cells during hypothermic storage depending on the preservation medium and ozone pretreatment

\begin{tabular}{ccccc}
\hline Storage & \multicolumn{2}{c}{ RBC in the Alsever's solution } & \multicolumn{2}{c}{ RBC in mannitol medium } \\
\cline { 2 - 5 } time, weeks & non-ozonated & ozonated & non-ozonated & ozonated \\
\hline 0 & $0.20 \pm 0.08$ & $0.32 \pm 0.14$ & $0.15 \pm 0.06$ & $0.22 \pm 0.14$ \\
1 & $4.84 \pm 0.33$ & $4.39 \pm 0.31$ & $3.61 \pm 0.28$ & $3.12 \pm 0.20$ \\
6 & $14.93 \pm 0.78$ & $11.40 \pm 0.66^{\#}$ & $8.47 \pm 0.64^{*}$ & $8.10 \pm 0.53^{*}$ \\
8 & $16.31 \pm 1.56$ & $12.09 \pm 0.86^{\#}$ & $13.21 \pm 0.88^{*}$ & $10.77 \pm 0.76^{\#}$ \\
12 & $38.52 \pm 1.92$ & $34.44 \pm 1.80^{\#}$ & $27.41 \pm 1.65^{*}$ & $23.39 \pm 1.31^{* \#}$ \\
\hline
\end{tabular}

Note: * - significant differences compared to the Alsever's solution $(\mathrm{P}<0.05),{ }^{*}$ significant differences compared to non-ozonated cells $(\mathrm{P}<0.05) ; \mathrm{n}=5$.

High deformability of erythrocytes when they pass through narrow vessels in the microcirculation process is considered to be mainly due to the viscoelastic properties of the plasma membrane of cells. Therefore, we investigated the osmotic fragility of ovine erythrocytes in different media in control and after hypothermic storage. It was found that with decreasing $\mathrm{NaCl}$ concentration, there was a sharp increase in the level of erythrocyte hemolysis (Fig. 1a-d) for all experimental groups that indicated very low osmotic stability of ovine erythrocytes. Nevertheless, the hypothermic storage of ovine red blood cells for 8 weeks did not significantly reduce their osmotic resistance. Subsequent erythrocyte storage for up to 12 weeks resulted in an increase in erythrocyte hemolysis in all studied groups if compared to 8 weeks of storage, and therefore a rise in osmotic fragility of ovine erythrocytes. In ozonated erythrocytes in the mannitol medium, these changes were less pronounced (Fig. 1d).

It can be noted comparing the osmotic properties of ovine erythrocytes with human ones (Fig. 2) that their osmotic fragility is higher. Nonozonated ovine erythrocytes in the Alsever's solution increases 50\% hemolysis level in a $0.57 \% \mathrm{NaCl}$ solution, and human red blood cells in a $0.47 \%$ solution. Hypothermic storage of human erythrocytes for up to 8 weeks led to a significant increase in their osmotic fragility, which indicates their lower resistance to hypothermic storage in comparison with ovine erythrocytes. Prolongation of erythrocyte storage up to 12 weeks resulted in an augmentation of erythrocyte hemolysis compared to 8 weeks in all studied groups, which indicated a further increase in their osmotic fragility. The obtained results demonstrated that the osmotic properties of ovine erythrocyte membranes were highly preserved during the hypothermic storage for up to 8 weeks, in contrast to human erythrocytes. 

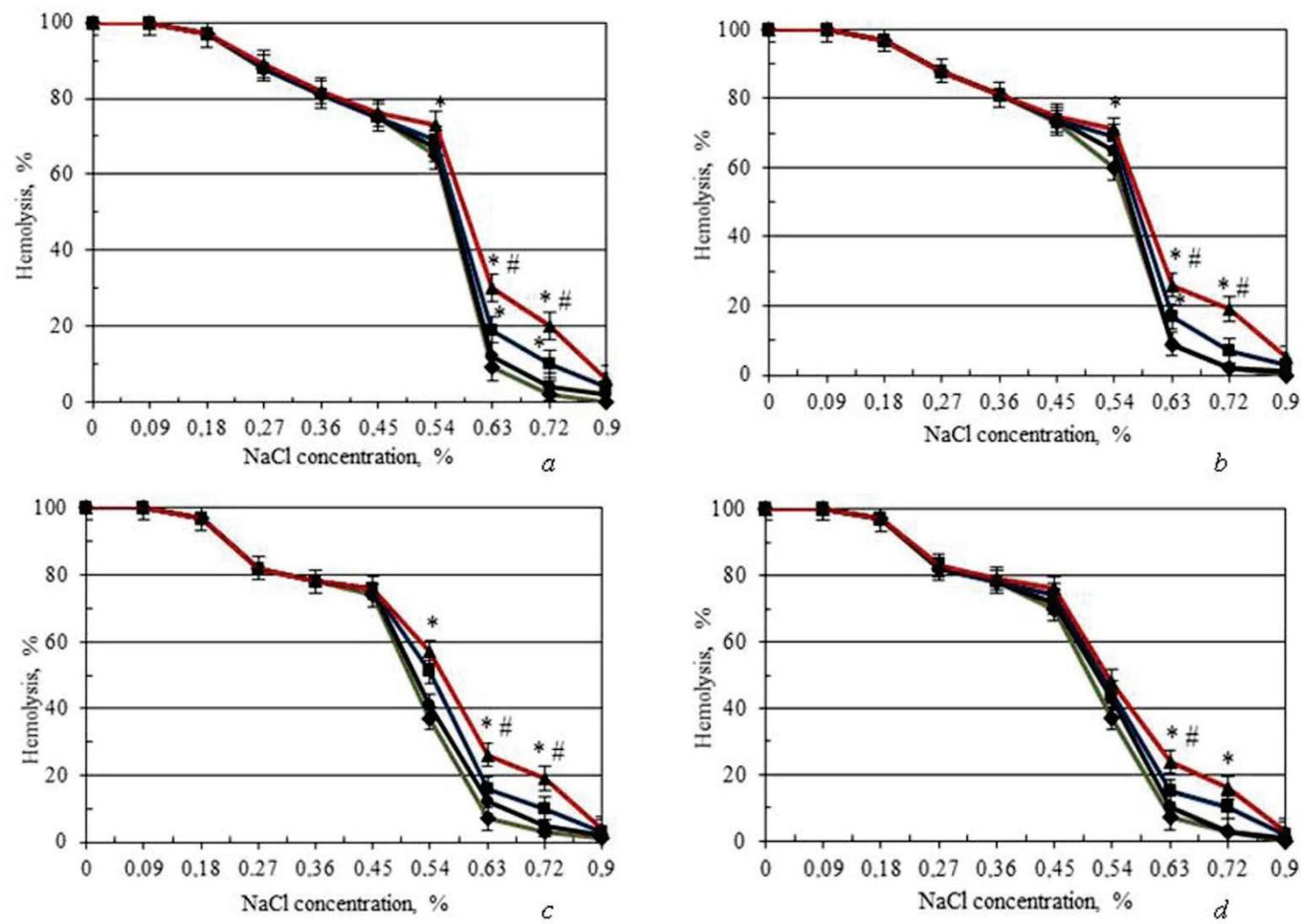

Fig. 1. Change in osmotic fragility of ovine erythrocytes during hypothermic storage:

$a$-non-ozonated in the Alsever's solution; $b$-ozonated in the Alsever's solution; $c$-non-ozonated in mannitol medium;

$d$ - ozonated in mannitol medium; green line - control; black line -4 weeks of storage; blue line -8 weeks of storage; red line - 12 weeks of storage; * - significant differences compared to control $(\mathrm{P}<0.05),{ }^{*}$ - significant differences compared to week 8 of storage $(\mathrm{P}<0.05) ; \mathrm{n}=5$
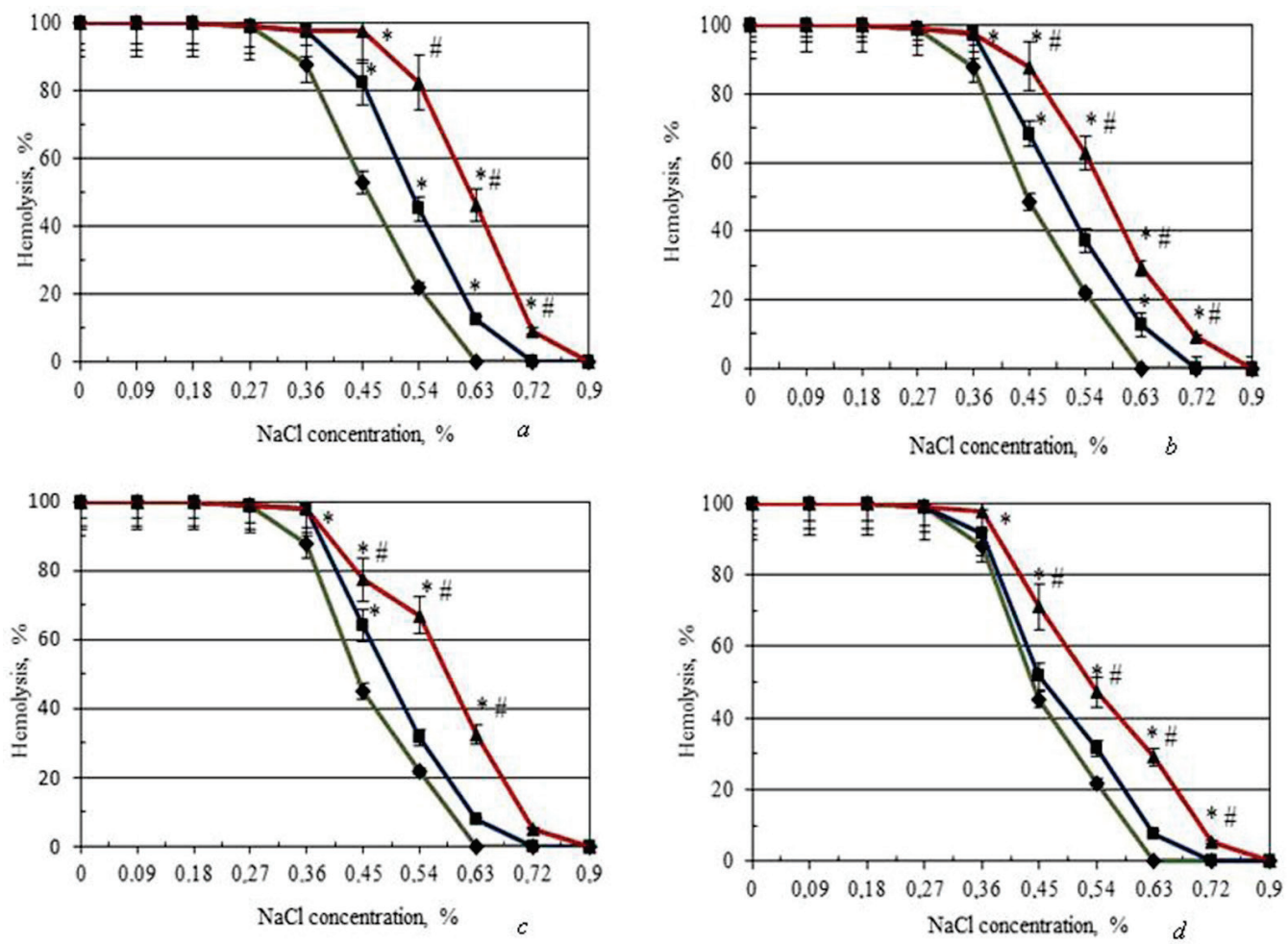

Fig. 2. Change in osmotic fragility of human erythrocytes during hypothermic storage:

$a$-non-ozonated in the Alsever's solution; $b$-ozonated in the Alsever's solution; $c$-non-ozonated in mannitol medium;

$d$-ozonated in mannitol medium; green line - control; blue line - 8 weeks of storage; red line - 12 weeks of storage; ${ }^{*}$ - significant differences compared to control $(\mathrm{P}<0.05),{ }^{\#}$ - significant differences compared to week 8 of storage $(\mathrm{P}<0.05) ; \mathrm{n}=5$

The density curves of the distribution of ovine and human erythrocytes according to the sphericity index were determined from experimental curves of osmotic fragility on the basis of a physico-mathematical model of hypotonic hemolysis in a solution of impermeable substance. It was revealed that the response of the erythrocyte populations to the stress caused by ozonation was manifested in an increase in the number of flattened cell forms (sphericity indices ranged 2.1-3.0) and a decrease in the number of shapes closer to spherical (sphericity indices ranged 1.0-1.3, Table 3, 4). It can be seen that more than $90 \%$ of ovine erythrocytes are in a narrow range of sphericity indices (1.3-1.7), which indicates a high homogeneity of the cell population. The distribution of control human erythrocytes according to the sphericity index shows a higher heterogeneity of the cell population. 
Table 3

Effect of ozonation and hypothermic storage on distribution of ovine erythrocytes by sphericity index

\begin{tabular}{cccccc}
\hline \multirow{2}{*}{ Conditions } & & \multicolumn{3}{c}{ Sphericity index } \\
\cline { 2 - 5 } & & $1.0-1.3$ & $1.3-1.7$ & $1.7-2.1$ & $2.1-3.0$ \\
\hline \multirow{2}{*}{ Control } & non-ozonated & $3.30 \pm 1.09$ & $92.13 \pm 3.68$ & $3.43 \pm 1.44$ & $1.14 \pm 0.49$ \\
6 weeks & ozonated & $1.32 \pm 0.41$ & $92.24 \pm 3.11$ & $1.76 \pm 0.68$ & $4.68 \pm 1.89^{*}$ \\
in the Alsever's solution & non-ozonated & $40.77 \pm 3.58^{*}$ & $54.65 \pm 2.93^{*}$ & $2.35 \pm 1.67$ & $2.23 \pm 0.78$ \\
6 weeks & ozonized & $46.01 \pm 4.12^{*}$ & $51.37 \pm 2.60^{*}$ & $1.19 \pm 0.56$ & $1.43 \pm 0.34$ \\
in mannitol medium & non-ozonated & $29.31 \pm 3.07^{*}$ & $69.24 \pm 11.13^{*}$ & $0.97 \pm 0.45$ & $0.48 \pm 0.21$ \\
8 weeks & ozonatzed & $23.83 \pm 2.12^{*}$ & $72.32 \pm 9.51^{*}$ & $2.05 \pm 0.99$ & $1.80 \pm 0.79$ \\
in the Alsever's solution & non-ozonated & $0.25 \pm 0.07^{*}$ & $46.63 \pm 3.66^{*}$ & $46.88 \pm 9.67^{*}$ & $6.25 \pm 4.11$ \\
8 weeks & ozonated & $0.12 \pm 0.09^{*}$ & $52.11 \pm 4.43^{*}$ & $45.77 \pm 7.23^{*}$ & $1.99 \pm 0.45$ \\
in mannitol medium & non-ozonated & $0^{*}$ & $1.51 \pm 0.36^{*}$ & $22.85 \pm 3.57^{*}$ & $75.64 \pm 8.32^{*}$ \\
\hline
\end{tabular}

Note: * - statistically significant differences compared to the control $(\mathrm{P}<0.05),{ }^{\sharp}$ - statistically significant differences compared to non-ozonated cells $(\mathrm{P}<0.05), \mathrm{n}=5$.

Table 4

Effect of ozonation and hypothermic storage on distribution of human erythrocytes by sphericity index

\begin{tabular}{|c|c|c|c|c|c|}
\hline \multirow{2}{*}{\multicolumn{2}{|c|}{ Conditions }} & \multicolumn{4}{|c|}{ Sphericity index } \\
\hline & & $1.0-1.3$ & $1.3-1.7$ & $1.7-2.1$ & $2.1-3.0$ \\
\hline \multirow[b]{2}{*}{ Control } & non-ozonatzed & $4.68 \pm 0.88$ & $35.17 \pm 1.89$ & $57.39 \pm 3.15$ & $2.76 \pm 0.51$ \\
\hline & ozonized & $0.15 \pm 0.08^{\#}$ & $33.79 \pm 2.23$ & $50.23 \pm 4.42$ & $15.83 \pm 3.20^{\#}$ \\
\hline 6 weeks & non-ozonated & $12.84 \pm 1.99^{*}$ & $83.33 \pm 3.12^{*}$ & $2.72 \pm 0.61^{*}$ & $1.11 \pm 0.24$ \\
\hline \multirow{2}{*}{$\begin{array}{c}\text { in the Alsever's solution } \\
6 \text { weeks }\end{array}$} & ozonized & $12.31 \pm 1.67^{*}$ & $82.12 \pm 3.67^{*}$ & $4.69 \pm 1.12^{*}$ & $0.88 \pm 0.32^{*}$ \\
\hline & non-ozonated & $4.82 \pm 0.57$ & $27.10 \pm 1.78^{*}$ & $64.87 \pm 4.21$ & $3.21 \pm 0.36$ \\
\hline in mannitol medium & ozonated & $3.45 \pm 0.79^{*}$ & $30.03 \pm 2.21$ & $62.61 \pm 4.02$ & $3.91 \pm 0.64^{*}$ \\
\hline \multirow{2}{*}{$\begin{array}{c}8 \text { weeks } \\
\text { in the Alsever's solution }\end{array}$} & non-ozonated & $42.13 \pm 3.11^{*}$ & $51.77 \pm 2.91^{*}$ & $2.27 \pm 0.31^{*}$ & $3.83 \pm 0.53$ \\
\hline & ozonated & $43.04 \pm 2.90^{*}$ & $51.59 \pm 3.32^{*}$ & $2.62 \pm 0.38^{*}$ & $2.75 \pm 0.67^{*}$ \\
\hline \multirow{2}{*}{$\begin{array}{c}8 \text { weeks } \\
\text { in mannitol medium }\end{array}$} & non-ozonated & $0.87 \pm 0.56^{*}$ & $2.89 \pm 1.20^{*}$ & $9.54 \pm 1.24^{*}$ & $86.7 \pm 5.45^{*}$ \\
\hline & ozonated & 0 & $11.79 \pm 3.13^{* \#}$ & $31.35 \pm 2.76^{* \#}$ & $56.86 \pm 6.18^{* \#}$ \\
\hline
\end{tabular}

Note: *-significant differences compared to the control $(\mathrm{P}<0.05),{ }^{*}$ - significant differences compared to non-ozonated cells $(\mathrm{P}<0.05), \mathrm{n}=5$.
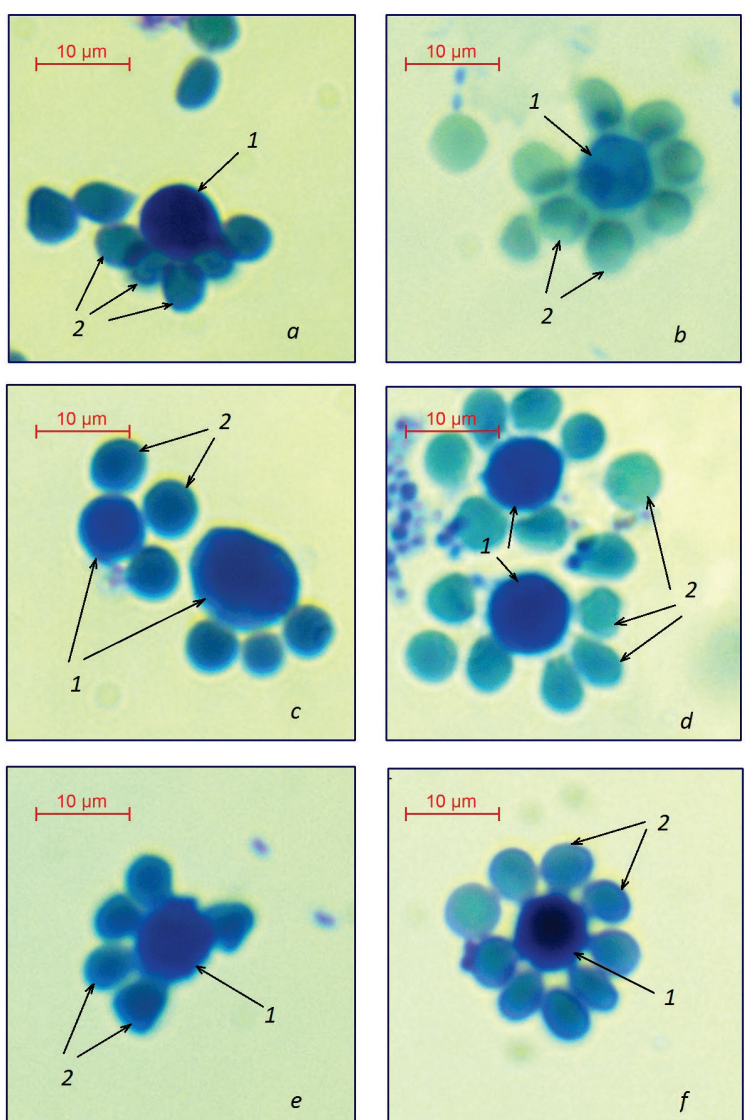

Fig. 3. Rosette formation of ovine erythrocytes with human lymphocytes: $a$-non-ozonated in the Alsever's solution; $b$-non-ozonated in mannitol medium; $c$-after hypothermic storage for 12 weeks in the Alsever's solution; $d$-after hypothermic storage for 12 weeks in the mannitol medium; $e$-ozonated, after hypothermic storage for 12 weeks in the Alsever's solution; $f$-ozonated, after hypothermic storage for 12 weeks in mannitol medium; 1 -human lymphocytes; 2 - ovine erythrocytes
Hypothermic storage of control and ozonated ovine erythrocytes for 6 weeks led to a change in their distribution according to the sphericity index (Table 3). In the Alsever's solution, the distribution shifted to the range of lower sphericity indices, which indicates a change in the shape of the cells to more spherical ones. In mannitol medium, the changes of cells' distribution are less pronounced. After hypothermic storage of human erythrocytes for 6 weeks in mannitol medium, the distribution of cells by the sphericity index is also close to that of the control, in contrast to the Alsever's solution (Table 4). An increase of storage time of human erythrocytes in the Alsever's solution of up to 8 weeks led to a further shift in the distribution density according to the sphericity index in the direction of spheroidization of cells; in mannitol medium there is an increase in the number of flattened cell shapes. In the group of ozonated cells in a mannitol medium, the distribution of cells is closer to the control than non-ozonated ones. Hypothermic storage of ovine erythrocytes up to 8 weeks led to a shift of the distribution density curves to a range of higher indices. For ozonized ovine erythrocytes, the distribution of cells according to the sphericity index was also closer to the control as compared to nonozonized ones. In order to quantify the major subpopulations of $\mathrm{T}$ and $\mathrm{B}$ lymphocytes for diagnosis purposes in practice the rosette formation methods are still used due to their low cost and simplicity. The spontaneous rosette formation is based on the fact that on the surface of all $\mathrm{T}$ lymphocytes there is a protein receptor for sheep erythrocytes. When $\mathrm{T}$ cells are mixed together with erythrocytes they form rosettes which can be separated from incapacitated ones. We evaluated the preservation of the ability of ovine erythrocytes to spontaneously form rosettes after hypothermic storage in the Alsever's solution and mannitol medium (Fig. 3). It was shown that even after 12 weeks of hypothermic storage ovine erythrocytes retain the ability to rosette formation with human lymphocytes (Fig. 3c-d). The ability of erythrocytes to spontaneously form rossettes in a mannitol medium after 12 weeks' storage (Fig. 3d) was not worse than control erythrocytes (Fig. 3b). The positive influence of ozonation on the preservation rate of this ability was revealed (Fig. 3e-f).

\section{Discussion}

Ovine erythrocytes have some of the lowest resistance among mammals to mechanical (Jikuya et al., 1998) and osmotic stresses (Matsuzava 
\& Ikarashi, 1979) and at the same time are among the smallest in size (only in goats are they smaller) (Adili \& Melizi, 2014). In this research we showed a high level of osmotic fragility, confirming the data of other researchers. However, their high state of preservation during hypothermic storage of up to two months, which we identified, was very surprising. Moreover, even after three months of storage, non-hemolyzed erythrocytes (and they are more than 70-80\%, depending on the medium and processing) retain the ability to form rosettes, although their osmotic fragility is significantly reduced. Such unique resistance to hypothermic storage can be associated with the surface-area-to volume ratio of a cell or with the peculiarities of the composition of membranes of ovine erythrocytes (they contain uniquely a lot of protein of the band 2.1-2.3) (Matei et al., 2000). Thus, ovine red blood cells have a high resistance to hypothermic storage, in contrast to human erythrocytes, although their lifetime in the bloodstream differs slightly.

Kisielewicz \& Self (2014) found that the pH of animals' blood was lowered during storage due to the accumulation of lactic and pyruvic acid, and contributed to a decrease in 2.3-diphosphoglycerate (2.3-DPG). The low level of 2.3-DPG causing the change of hemoglobin affinity for oxygen after transfusion, affected the tissue oxygenation, morbidity and mortality in animals. Hoffmann \& Viebahn (2001) showed that the treatment of erythrocytes with ozone could slow down the decrease in 2.3DPG levels in hypothermic storage, which might ensure a better prognosis during transfusion. The positive effect of ozone on ovine erythrocyte preservation can be associated with the level 2.3-DPG in cells. Moreover, there was a correlation between the rates of hemolysis of sheep erythrocytes, their osmotic fragility and the ability to form rosettes with human T lymphocytes assessed in our work. The ozone treatment provided the reduced rate of hemolysis, increased the osmotic resistance of erythrocytes during hypothermic storage, and maintained their ability to form rosettes. Thus, the use of ozone can be very promising for the creation of animals' blood banks, in particular for erythrocytes of sheep.

\section{Conclusions}

Ovine erythrocytes have lower osmotic resistance than human red blood cells, but their osmotic properties do not change significantly during the first 8 weeks of hypothermic storage. Storage of ovine and human erythrocytes longer than 8 weeks leads to a sharp increase in hemolysis and osmotic fragility. Ozonation treatment of red blood cells increases their preservation rate during prolonged hypothermic storage. The effect of ozonation on preservation of red blood cells during hypothermic storage depends on the protective medium. Ovine erythrocytes retain the ability to form rosettes with human $\mathrm{T}$ lymphocytes after hypothermic storage for up to 12 weeks. Treatment of ovine erythrocytes with ozone and their storage in a mannitol medium improved preservation of ability to spontaneously form rosettes.

\section{References}

Adili, N., \& Melizi, M. (2014). Preliminary study of the influence of red blood cells morphometry on the species determinism of domestic animals. Veterinary World, 7(4), 219-223.

Baskurt, O. K., Hardeman, M. R., \& Rampling, M. W. (2007). Handbook of hemorheology and hemodynamics. IOS press, Amsterdam.

Bell, G. (2006). Blood transfusions in cattle. Livestock, 11(3), $39-43$.

Chandler, K., Fitzpatrick, J., Mellor, D., Milne, M., \& Fishwick, G. (1998). Intraperitoneal administration of whole blood as a treatment for anaemia in lambs. The Veterinary Record, 142(7), 175-176.

Davidow, B. (2013). Transfusion medicine in small animals. Small Animal Practice, 43(4), 735-756.

Durham, A. E. (1996). Blood and plasma transfusion in the horse. Equine Veterinary Education, 8(1), 8-12.

Fung, Y. L., Simonova, G., \& Tung, J. P. (2016). Lessons from sheep models of transfusion. ISBT Science Series, 11(2), 73-78.

Gordiyenko, O. I., Gordiyenko, Y. E., \& Makedonska, V. O. (2004). Estimation of erythrocyte population state by the spherical index distribution. Bioelectrochemistry, 62, 119-122.
Jikuya, T., Tsutsui, T., Shigeta, O., Sankai, Y., \& Mitsui, T. (1998). Species differences in erythrocyte mechanical fragility: Comparison of human, bovine, and ovine cells. American Society for Artificial Internal Organs Journal, 44(5), 452- 455.

Kisielewicz, C., \& Self, I. A. (2014). Canine and feline blood transfusions: Controversies and recent advances in administration practices. Veterinary Anaesthesia and Analgesia, 41(3), 233-242.

Klaus, J. (1990). Lymphocytes: Methods. Mir, Moskow (in Russian).

Kumar, R. (2017). Blood transfusion in veterinary medicine. Hematology and Transfusion International Journal, 4(4), 116-122.

Ladics, G. S. (2007). Primary immune response to sheep red blood cells (SRBC) as the conventional T-cell dependent antibody response (TDAR) test. Journal of Immunotoxicology, 4(2), 149-152.

Le Gal, A., Thomas, E. K., \& Humm, K. R. (2020). Xenotransfusion of canine blood to cats: A review of 49 cases and their outcome. Journal of Small Animal Practice, 61(3), 145-208.

Lomako, V. V., Kovalenko, I. F., \& Shilo, A. V. (2012). Peripheral blood erythrocytes at various types of hypothermia of homoiothermal organism problems of cryobiology, 22 (4), 389-397.

Martinez-Sogues, L, Blois, S. L., Manzanilla, E. G., Abrams-Ogg, A. O., \& Cosentino, P. (2020). Exploration of risk factors for non-survival and for transfusionassociated complications in cats receiving red cell transfusions: 450 cases (2009 to 2017). Journal of Small Animal Practice, 61(3), 177-184.

Matei, H., Frentescu, L., \& Benga, G. (2000). Comparative studies of the protein composition of red blood cell membranes from eight mammalian species. Journal of Cellular and Molecular Medicine, 4(4), 270-276.

Matsuzawa, T., \& Ikarashi, Y. (1979). Haemolysis of various mammalian erythrocytes in sodium chloride, glucose and phosphate-buffer solutions. Laboratory Animals, 13(4), 329-331.

McAllister, E. J., Apgar, J. R., Leung, C. R., Rickert, R. C., \& Jellusova, J. (2017). New methods to analyze B cell immune responses to thymus-dependent antigen sheep red blood cells. The Journal of Immunology, 199(8), 2998-3003.

Ostras, D. A., Lutsenko, D. G., Lomako, V. V., Kovalenko, I. F., \& Shylo, O. V. (2019). Changes in erythrocytes osmotic fragility and sphericity index in rat's of different ages under cold exposures. Problems of Cryobiology and Cryomedicine, 29(2), 172.

Park, H. J., Lee, S. Y., Ji, M., Kim, K., Son, Y. H., Jang, S., \& Park, Y. K. (2016). Measuring cell surface area and deformability of individual human red blood cells over blood storage using quantitative phase imaging. Scientific Reports, 6 , 34257.

Ryskina, E. A., \& Gilmiyarova, F. N. (2015). Group antigens of various animals. RUDN Journal of Agronomy and Animal Industries, 1, 25-34 (in Russian).

Saganuwan, S. A. (2019). Effects of therapeutic and toxic agents on erythrocytes of different species of animals. In: Tombak, A. (Ed.). Erythrocyte. IntechOpen, London.

Saritha, G., Haritha, G. S., Kumari, K. N., \& Sundar, N. S. (2016). Blood transfusion in a calf with life-threatening anemia. IOSR Journal of Agriculture and Veterinary Science, 9(5), 69-70.

Shinbrot, T. (2019). Biomedical fluid dynamics: Flow and form. Oxford University Press, Oxford.

Simonova, G., Tung, J. P., Fraser, J. F., Do, H. L., Staib, A., Chew, M. S., Dunster, K. R., Glenister, K. M., Jackson, D. E., \& Fung, Y. L. (2014). A comprehensive ovine model of blood transfusion. Vox Sanguinis, 106(2), 153-160.

Sousa, R. S., Minervino, A. H. H., Araújo, C. A. S. C., Rodrigues, F. A. M. L., Oliveira, F. L. C., Mori, C. S., Zaminhan, J. L. R., Moreira, T. R., Sousa, I. K. F., Ortolani, E. L., \& Barrêto Júnior, R. A. (2014). Clinical response and transfusion reactions of sheep subjected to single homologous blood transfusion. The Scientific World Journal, 2014, 734397.

Wain, E. B., \& Redpath, J. A. (1985). Blood transfusion as a treatment of anaemia in lambs. The Veterinary Record, 116(19), 527-527.

Wardrop, K. J., Reine, N., Birkenheuer, A., Hale, A., Hohenhaus, A., Crawford, C., \& Lappin, M. R. (2005). Canine and feline blood donor screening for infectious disease. Journal of Veterinary Internal Medicine, 19(1), 135-142.

Webb, G. (2019). Canine and feline blood transfusions. The Veterinary Nurse, 10(3), $139-145$.

Windberger, U., Pöschl, C., Peters, S., Huber, J., \& Van den Hoven, R. (2017). Whole blood of mammalian species in the oscillating shear field: Influence of erythrocyte aggregation. In: IOP Conference Series: Materials Science and Engineering, 175(1), 012005 .

Wybran, J., Carr, M. C., \& Fudenberg, H. H. (1972). The human rosette-forming cell as a marker of a population of thymus-derived cells. The Journal of Clinical Investigation, 51(10), 2537-2543.

Yagi, K., \& Holowaychuk, M. (2016). Manual of veterinary transfusion medicine and blood banking. Wiley Blackwell. 